\title{
Video Article \\ Measuring Biophysical and Psychological Stress Levels Following Visitation to Three Locations with Differing Levels of Nature
}

\author{
Yun Chang ${ }^{1}$, Alan Ewert ${ }^{2}$, Lisa M. Kamendulis ${ }^{3}$, Barbara A. Hocevar ${ }^{3}$ \\ ${ }^{1}$ School of Kinesiology and Recreation, Illinois State University \\ ${ }^{2}$ Department of Recreation, Park, and Tourism Studies, Indiana University \\ ${ }^{3}$ Department pf Environmental and Occupational Health, School of Public Health-Bloomington, Indiana University
}

Correspondence to: Yun Chang at ychan12@ilstu.edu

URL: https://www.jove.com/video/59272

DOI: doi:10.3791/59272

Keywords: Behavior, Issue 148, a-amylase, biomarkers, multiple methods, natural environments, restoration, salivary cortisol, stress

Date Published: 6/19/2019

Citation: Chang, Y., Ewert, A., Kamendulis, L.M., Hocevar, B.A. Measuring Biophysical and Psychological Stress Levels Following Visitation to Three Locations with Differing Levels of Nature. J. Vis. Exp. (148), e59272, doi:10.3791/59272 (2019).

\section{Abstract}

Visitation to natural environments has been linked to psychological stress reduction. Although most stress-related research has relied on selfreport formats, a growing number of studies now incorporate biological stress-related hormones and catalysts, such as cortisol and $\alpha$-amylase, to measure levels of stress. Presented here is a protocol to examine the effects on levels of biophysical and psychological stress following visitation to three different locations with differing levels of nature. Biophysical and self-reported psychological stress levels are measured immediately upon entering the selected locations and just prior to the visitors leaving the site. Using a "drool" method, the biophysical measure consists of 1-2 $\mathrm{mL}$ samples of saliva provided by study subjects upon entry to one of three study locations. As prescribed by extant literature, the saliva is collected within a 45 minute time frame following the end of the visitor's engagement at the location. Following saliva collection, the samples are labeled and transported to a biological lab. Cortisol is the biophysical variable of interest in this study and measured using an ELISA process with a TECAN plate reader. To measure self-reported stress, the Perceived Stress Questionnaire (PSQ), which reports levels of worry, tension, joy, and perceived demands. Data are collected at all three sites in the late afternoon through early evening. When compared across all three settings, stress levels, as measured by both the biological markers and self-reports, are significantly lower after visitation to the most natural setting.

\section{Video Link}

The video component of this article can be found at https://www.jove.com/video/59272/

\section{Introduction}

Elevated stress levels have long been linked to many serious health conditions such as heart disease, obesity, and psychological disorders ${ }^{1,2,3}$ A growing body of research suggests that close proximity or visitation to natural settings such as park and non-developed landscapes can have a remarkable effect on psychological well-being and decreased levels of stress ${ }^{1,4,5,6,7,8,9,10}$. Explanations for the effects of natural settings and stress levels have included the following: (1) natural settings provide venues for physical activity ${ }^{8,11}$ and (2) visitors to natural environments have the ability to focus on more non-task thought processes, thereby leading to a reduction in attention fatigue ${ }^{2}$. To determine the effects of nature on stress reduction, this study utilizes a self-report of psychological stress (PSQ) and two saliva-based biomarkers, cortisol and $\alpha$-amylase, after visitation to three different recreation sites. These locations vary among their levels of "naturalness" and include a wilderness-type setting, municipal park, and local fitness and recreation facility.

This study aims to address the following research questions: $\left(R Q_{1}\right)$ Are there differences in levels of biophysical stress measured by salivary cortisol and $\alpha$-amylase when compared across all three sites (i.e., natural, semi-natural, built)? $\left(R Q_{2}\right)$ Are there differences in levels of psychological stress measured by the PSQ (manifested by four constructs: demands, worries, tension, and joy) when compared across all three sites (i.e., natural, semi-natural, built)?

\section{Protocol}

This study follows the policies and guidelines of Human Research Protection Program of Indiana University Institutional Review Board

\section{Location selection}

1. Select the number of sites $(n)$ based on different levels of nature.

NOTE: We chose three sites for our work. Using a continuum based on levels of "naturalness", Site A was considered the most natural and is comprised of approximately 1,200 acres of wooded ridges bordering a lake and set within a deciduous forest. The most common activities 
include walking and wildlife watching. Site B was a 33-acre municipal park featuring walking paths, places for gatherings, playgrounds, and open field space for causal recreational activities. Site $\mathrm{C}$ was an urban, indoor exercise facility with the lowest level of naturalness. All three sites are in relatively close proximity to a medium-size city (estimated population of 46,000 people) in the midwestern United States.

\section{Participant screening and preparation}

1. Complete participant recruitment before subjects engage in recreational experiences. NOTE: For Sites A and B, subjects were approached by the researcher in the parking lot of the park entrance. For Site C, subjects were approached at the front desk of the indoor exercise facility. To control for differences in activity type, subjects recruited for both Sites A and B were predominately hiking, while for Site $C$ the primary activity was running or walking on the indoor track.

2. During recruitment, remind subjects to comply with the guidelines related to collecting salivary cortisol samples. They must not eat or drink 10 min before providing saliva samples.

NOTE: It should be noted that in this case, the saliva samples were not normalized for volume or dilution (concentration) - i.e. whether the participants were adequately hydrated and/or did hydration change between the sample collections.

3. Give red armbands (or equivalently noticeable clothing) to each participant to allow for easy identification when emerging at the end of their engagements.

4. Assign a 30-40 min time allotment so that all subjects across the three sites spend a similar amount of time in each site. Exclude subjects who spend substantially more or less time than the rest of the sample.

5. Keep the type of activity engaged consistent.

NOTE: In this case, the "medium" activity was walking or hiking. Subjects who engaged in activities substantially different from walking or hiking were excluded from the sample. For example, subjects who were fishing, picnicking, or weight lifting were not included in the respective samples.

\section{Conditions and experimental design}

1. Use a quasi-experimental pre-test/post-test design.

2. After identifying subjects and upon their agreeing to participate, ask each subject to read and sign an IRB form explaining the voluntary nature, purpose, and procedures of the study.

3. Following this process, give subjects a piece of clothing (e.g., red armband) for future identification and obtain physiological and psychological measures of stress levels (i.e., PSQ, $1-2 \mathrm{~mL}$ of saliva that is spit or drooled into a test tube). Collect samples from the late afternoon to early evening.

NOTE: These data were collected by researchers both 1) just before subjects entered the site and 2) immediately upon ending visitation to the site.

\section{Saliva samples}

1. To avoid sample dilution, ask subjects not to eat, drink, or rinse mouths 10 min before providing saliva samples.

2. Ask subjects to provide $1-2 \mathrm{~mL}$ saliva samples (excluding foam) just prior to the recreational experience and immediately following conclusion of the experience.

3. Collect saliva samples using a passive drool method:

1. Provide subjects with a 2 in plastic drinking straw to drool into a $2 \mathrm{~mL}$ cryovial (see Table of Materials).

2. Instruct subjects to allow saliva to pool in their mouths, then drool down the straw and into the cryovial. According to the Saliva Collection and Handling Advice (2011), $1 \mathrm{~mL}$ (excluding foam) is adequate for most tests.

3. Label samples with an assigned 3-digit ID number (i.e., 001) and letter to indicate the timing of data collection (i.e., A represents pretest, $\mathrm{B}$ represents post-test; where 001A represents the saliva sample provided by participant 001 before conducting the recreational experience).

4. After the sample is collected and labeled, it should then be stored frozen temporarily in a steroid foam box full of dry ice for no more than $2 \mathrm{~h}$.

5. Transport the marked samples to a laboratory and store at $-80^{\circ} \mathrm{C}$ until analyzed.

\section{Quantification of $\alpha$-amylase}

NOTE: In this assay, $\alpha$-amylase hydrolyzes 2-chloro-p-nitrophenyl- $\alpha$-D-maltotrioside to 2-chloro-nitrophenol and forms glucose, 2-chloro-pnitrophenyl- $\alpha$-D-maltoside, maltotriose, and glucose. The reaction is monitored at an absorbance of $405 \mathrm{~nm}$, which corresponds to $\alpha$-amylase activity in the sample. This assay demonstrates linearity between 0 and $2000 \mathrm{U} / \mathrm{L}$.

1. Materials

1. Used a liquid amylase reagent set (see Table of Materials) to quantify $\alpha$-amylase in saliva samples. All reagents are provided as ready-to-use liquids and stored at $0-4{ }^{\circ} \mathrm{C}$.

2. Use a multi-mode reader (see Table of Materials) capable of reading an optical density at $405 \mathrm{~nm}$ with a temperature controlled to 37 ${ }^{\circ} \mathrm{C}$ during the assay.

2. Analysis

1. Thaw the samples on ice prior to analysis.

2. Dilute samples $1: 10$ with $1 \times$ PBS $(10 \mu \mathrm{L}$ of saliva $+90 \mu \mathrm{L}$ of PBS).

3. Analyze each sample in duplicate.

4. Equilibrate the amylase reagent to $20-25^{\circ} \mathrm{C}$ for at least $30 \mathrm{~min}$. 
5. Add $0.1 \mathrm{~mL}$ of amylase reagent to a 96 well microplate for each sample.

6. Pre-incubate the microplate at $37^{\circ} \mathrm{C}$ for a minimum of $5 \mathrm{~min}$.

7. Add $2.5 \mu \mathrm{L}$ of the sample to the amylase reagent.

8. Take an initial reading after $60 \mathrm{~s}$.

9. Continue readings every $60 \mathrm{~s}$ for an additional $2 \mathrm{~min}$.

10. Calculate the mean absorbance difference per minute $(\triangle A b s / \mathrm{min})$.

\section{Calculation}

1. To calculate amylase activity, use the following formula:

$\frac{\triangle A b s / \min * T V * 1000}{M M A * S V * L P}=\frac{U}{L} \alpha$ amylase in saliva

Where $\triangle A b s / \mathrm{min}=$ changes in absorbance difference per minute; $T V=$ total assay volume $(0.1025 \mathrm{~mL}) ;{ }^{*} 1000=$ conversion of $\mathrm{U} / \mathrm{mL}$ to $\mathrm{U} / \mathrm{L} ; M M A=$ millimolar absorptivity of 2-chloro-p-nitrophenol = 12.9; $S V=$ sample volume $(0.0025 \mathrm{~mL})$; and $L P=$ light path $(1 \mathrm{~cm})$. Substituting gives:

$\frac{\Delta A b s / \min * 0.1025 * 1000}{12.9 * 0.025 * 1.0}=\frac{\Delta A b s}{\min } * 3178=\frac{U}{L}$ a amylase

Therefore, multiply the $\triangle A b s / \mathrm{min}$ by $3178 \mathrm{x}$ the dilution factor (10) to obtain amylase in U/L.

2. For samples above $2000 \mathrm{U} / \mathrm{L}$ (assay linearity) dilute further (at least $2 x$ using PBS) and re-assay, then multiply the $\alpha$-amylase result by the additional dilution factor.

\section{Quantification of cortisol}

NOTE: In this assay, free cortisol is quantified in saliva using a cortisol standard curve. Standards and diluted samples are added to a microtiter plate that is pre-coated with an antibody. A cortisol-peroxidase conjugate is added to the wells, followed by addition of a monoclonal antibody to cortisol. The amount of cortisol/peroxidase conjugate binding decreases as the concentration of cortisol in the sample increases.

\section{Materials}

1. Use a Cortisol Enzyme Immunoassay Kit (see Table of Materials) to quantify cortisol in saliva samples. All reagents needed to perform this assay are included in the kit. All components of the kit are stored at $0-4{ }^{\circ} \mathrm{C}$ prior to reaching the expiration date.

2. Use a spectrophotometer able to read an optical density (OD) at $450 \mathrm{~nm}$ (see Table of Materials), as well as software capable of using $\mathrm{OD}$ recordings from the plate reader to perform four-parameter logistic curve (4 PLC) fitting.

\section{Reagent preparation}

1. Allow all reagents to equilibrate to $20-25^{\circ} \mathrm{C}$ for a minimum of $30 \mathrm{~min}$.

2. Dilute the cortisol assay buffer 1:5 using deionized water.

3. Dilute the wash buffer 1:20 using deionized water.

NOTE: Assay and wash buffers are stable for 3 months when stored at $0-4{ }^{\circ} \mathrm{C}$.

\section{Sample preparation}

1. Thaw the samples on ice prior to analysis.

2. Dilute samples $1: 10$ with cortisol assay buffer ( $20 \mu \mathrm{L}$ of saliva $+180 \mu \mathrm{L}$ of buffer) and use within $2 \mathrm{~h}$ of preparation.

\section{Preparation of standards}

1. Label glass test tubes \#1-\#7.

2. Pipet $225 \mu \mathrm{L}$ of assay buffer into tube \#1 and $125 \mu \mathrm{L}$ of buffer into tubes \#2-\#7.

3. Add $25 \mu \mathrm{L}$ of the cortisol stock solution to tube \#1 and vortex.

4. Remove $125 \mu \mathrm{L}$ of buffer from tube \#1 and add it to tube \#2, then vortex.

5. Repeat the serial dilutions for tubes \#3-\#7.

NOTE: The final cortisol concentrations of each standard is shown in Table 1.

6. Standards must be used within $2 \mathrm{~h}$ of preparation.

\begin{tabular}{|c|c|c|c|c|c|c|c|}
\hline Standard & $\# 1$ & \#2 & $\# 3$ & $\# 4$ & \#5 & \#6 & $\# 7$ \\
\hline $\begin{array}{l}\text { Assay Buffer } \\
\text { Volume }(\mu \mathrm{L})\end{array}$ & 225 & 125 & 125 & 125 & 125 & 125 & 125 \\
\hline Addition & Stock Std & $\# 1$ & $\# 2$ & $\# 3$ & $\# 4$ & $\# 5$ & $\# 6$ \\
\hline \begin{tabular}{|l} 
Volume of \\
Addition $(\mu \mathrm{L})$
\end{tabular} & 25 & 125 & 125 & 125 & 125 & 125 & 125 \\
\hline \begin{tabular}{|l|} 
Final \\
Concentration \\
$(\mathrm{pg} / \mathrm{mL})$
\end{tabular} & 3200 & 1600 & 800 & 400 & 200 & 100 & 50 \\
\hline
\end{tabular}

Table 1: Standard curve preparation table.

\section{Analysis}

1. Use the plate layout below in Figure $\mathbf{1}$ as a guide to set up the microplate.

2. It is recommended to use a multichannel pipette for addition of reagents. 
3. Add $50 \mu \mathrm{L}$ of samples or standards into the appropriate number of wells in the plate. Samples and standards should be run in duplicate.

4. Add assay buffer $(75 \mu \mathrm{L})$ into the non-specific binding (NSB) wells.

5. Add assay buffer $(50 \mu \mathrm{L})$ into the maximum binding (B0) wells and zero standard (blank) wells.

6. Add $25 \mu \mathrm{L}$ of the cortisol conjugate to each well.

7. Add $25 \mu \mathrm{L}$ of the cortisol antibody to each well, except for the NSB wells.

8. Gently tap the side of the plate to mix the reagents.

9. Cover with the plate sealer and shake at room temperature (RT) at $20-25^{\circ} \mathrm{C}$ for $1 \mathrm{~h}$.

10. Remove the well contents and rinse each well $4 x$ with wash buffer $(300 \mathrm{Ml})$. Tap the plate to dry on absorbent towels between washes.

11. Add the TMB substrate to each well $(100 \mu \mathrm{L})$.

12. Incubate the plate at $20-25^{\circ} \mathrm{C}$ for 30 min without shaking.

13. Add stop solution to each well $(50 \mu \mathrm{L})$.

14. Read the optical density in each well of the microplate at $450 \mathrm{~nm}$.

15. Average the optical densities for each standard, then sample and subtract the mean optical density for the NSB wells.

16. Calculate the $\%$ bound (B/B0) for all samples using maximum binding (B0) controls.

17. Create a standard curve using software capable of four-parameter logistic regression curve fitting, calculated from the \%B/B0 curve.

18. Multiply the result by the dilution factor (10) to obtain cortisol values in $\mathrm{pg} / \mathrm{mL}$.

19. Samples with optical densities falling above the highest standard should be further diluted with assay buffer and re-assayed, then the result should be multiplied by the additional dilution factor.

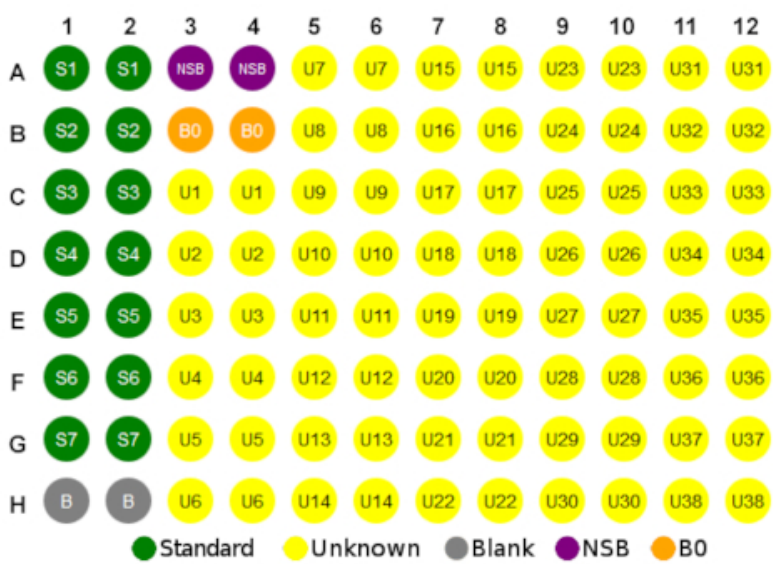

Figure 1: Example plate layout. Please click here to view a larger version of this figure.

\section{Psychological measurement (Perceived Stress Questionnaire)}

1. Measure psychological levels of subjects using the PSQ published by Fliege et al. ${ }^{13}$, which includes four factors (worry, tension, tension, joy) and utilizes 20 items.

2. Ask the subject to fill out the PSQ just prior to their recreational experience and immediately following conclusion of the experience.

3. Tag questionnaires with a 3-digit ID number identical to each subject's biophysiological level of stress.

\section{Representative Results}

\section{Sample Description}

Utilizing a quota sampling technique, this study recruited 35 visitors from each of the three sites. In total, 105 subjects were recruited in this study, including 63 males and 42 females. Average ages of visitors recruited from three different sites were 25.9 years (Site A), 37.2 years (Site $B$ ), and 28.8 years (Site C). Frequencies of subjects' visitation to the selected three sites were also recorded. For Site A and Site C, the majority of subjects visited this site one to three times per week. For subjects at Site B, their frequency of visitation was equally split between one to three times per week and more than three times per week.

Biophysical and psychological stress indicators. Biophysical measures of cortisol and $\alpha$-amylase levels were utilized to identify changes in levels of physiological stress. Changes in psychological stress were identified through the PSQ instrument.

\section{Effects of Recreational Site Visitation on Cortisol Levels and $\alpha$-amylase Levels}

The first research question asked if there would be a difference in levels of cortisol and a-amylase as a function of type of site (e.g., level of nature). A paired sample $t$-test resulted in a significant decrease in salivary cortisol after visiting Site $\mathrm{A}$ (natural setting) $\left[t_{31}=3.26, p<.01\right.$, see Figure 2]. No significant changes in levels of cortisol were observed in Site B and C. When comparing subjects changes in their cortisol levels across all three sites, the results of the ANOVA test indicated that different sites (levels of nature) did not have an overall significant impact on subjects changes in cortisol levels $\left[F_{(2,95)}=1.86, p=0.16\right]$ with small effect sizes $(0.01-0.04)$. 
Measurement of changes in levels of stress (pre/post visitation) using levels of $\alpha$-amylase resulted in mixed findings among the three study locations. Following the use paired sample $t$-tests indicate significant increases in levels of $\alpha$-amylase after visiting Site $C\left[t_{34}=2.79, p<.01\right.$. Statistical differences were not observed after visitation to Sites A or Site B. (See Figure 3). Analysis using ANOVA techniques indicated a main effect of location with different locations had a significant impact on subjects changes in $\alpha$-amylase levels $\left[F_{(2,101)}=3.36, p<0.05\right]$. Using Scheffe post-hoc analysis, $\alpha$-amylase levels were significantly higher after visiting Site $C$ when compared with Site $B$. There was a lack of significant differences on levels of $\alpha$-amylase when comparing visitors to Site A and Site B, or between Site A and Site C. Effect sizes of (0.03-0.01) were found and determined to be small.

\section{Effects of Recreational Site Visitation on Psychological Stress Levels}

Paired sample t-tests were applied to compare pre- and post-visit of levels of psychological stress among the three sites, respectively. As shown in Figure 4 and 5, after visitation to the three locations, significant decreases on the factors of demands and worry $(p<0.01)$ were observed. No significant changes in the factor, tension, was observed for any of the three locations. (See Figure 6). Significant increases were reported at Sites A and Site B for the factor, joy. No significant change was observed for visitors to Site C (see Figure 7).

To determine whether which of the three site is more efficacious in reducing subjects levels of psychological stress, the result of ANOVA tests indicated significant differences among the three sites $(p<0.01)$ with post-hoc analysis using the Scheffe's Method, reporting significant increases in levels of joy after visiting Site A, compared to visitors visiting Sites B and Site C. No differences were found in changes in levels of demands, worries, and tension $(p=0.27)$ across three locations.

In summary, visitors to Site A (most natural) reported significant decreased levels of cortisol; suggesting a reduction in biological stress levels. In addition, as measured by the PSQ, significant decreases in the psychological factors of demands and worries, and a significant increase in the level of joy were observed in visitors to Site A. Visitors to Site B (semi-natural) reported decreases in levels of demands and worries, and an increased level of joy. C (built environment) two decreases in level of demands and worries were reported. Interestingly, levels of $\alpha$-amylase increased significantly after visitation to Site $\mathrm{C}$. Further research is warranted to examining the influence of potential factors, such as specific activity or social surrounding, particularly on the catalyst, $\alpha$-amylase.

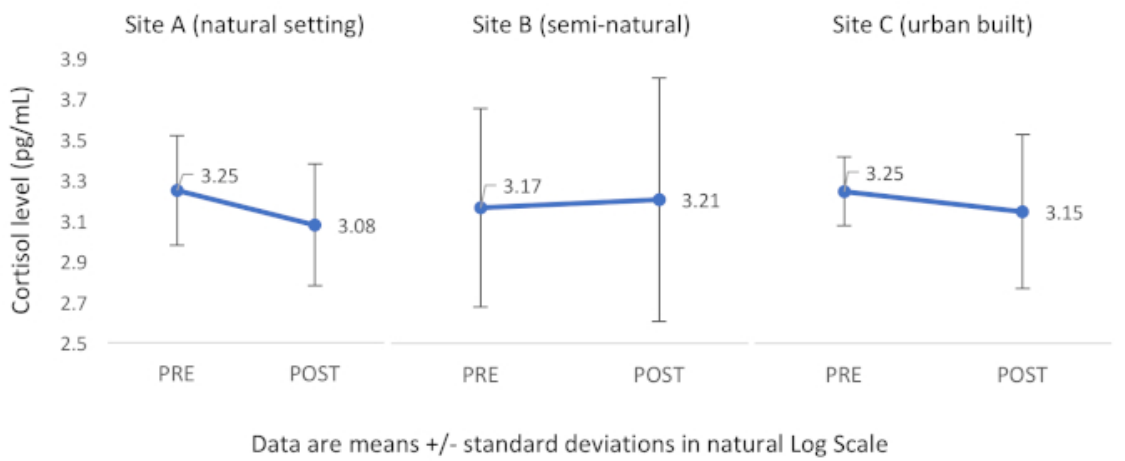

Figure 2: Changes in levels of cortisol after visiting three different sites. This figure shows subjects' cortisol levels measured before and after visiting three different sites representing different levels of nature: Sites A, B, and C. Data are presented as mean \pm SD in natural log scale. Please click here to view a larger version of this figure.

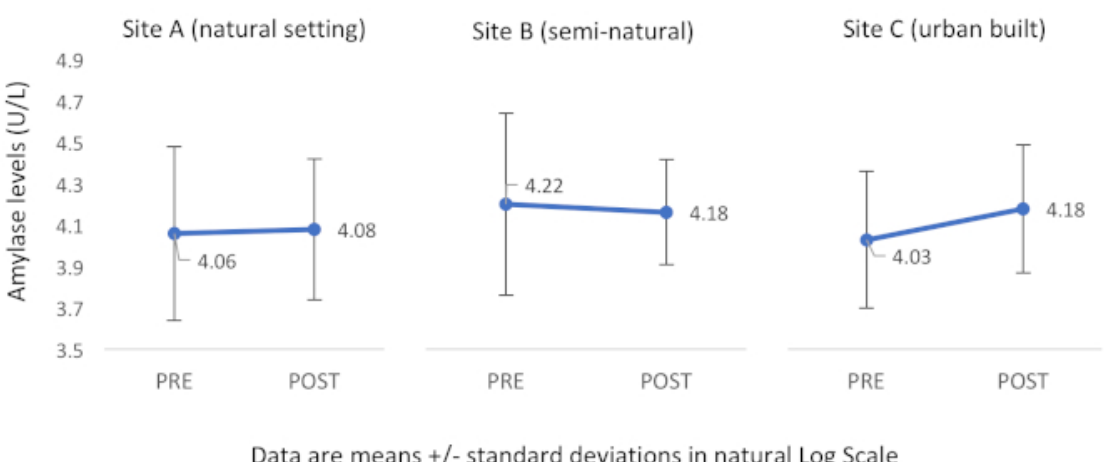

Figure 3: Changes in levels of amylase after visiting three different sites. This figure shows subjects amylase levels measured before and after visiting Sites $A, B$, and $C$. Data are presented as mean $\pm S D$ in natural log scale. Please click here to view a larger version of this figure. 


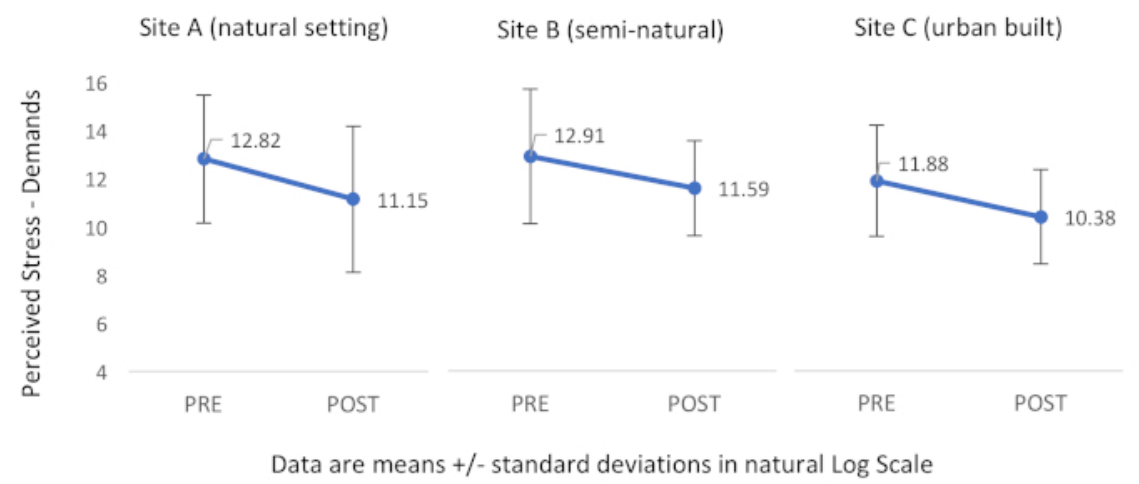

Figure 4: Changes in perceived levels of demands after visiting three different sites. This figure shows subjects' levels of demands decreased after visiting Sites A, B, and C. Data are presented as mean \pm SD. Please click here to view a larger version of this figure.

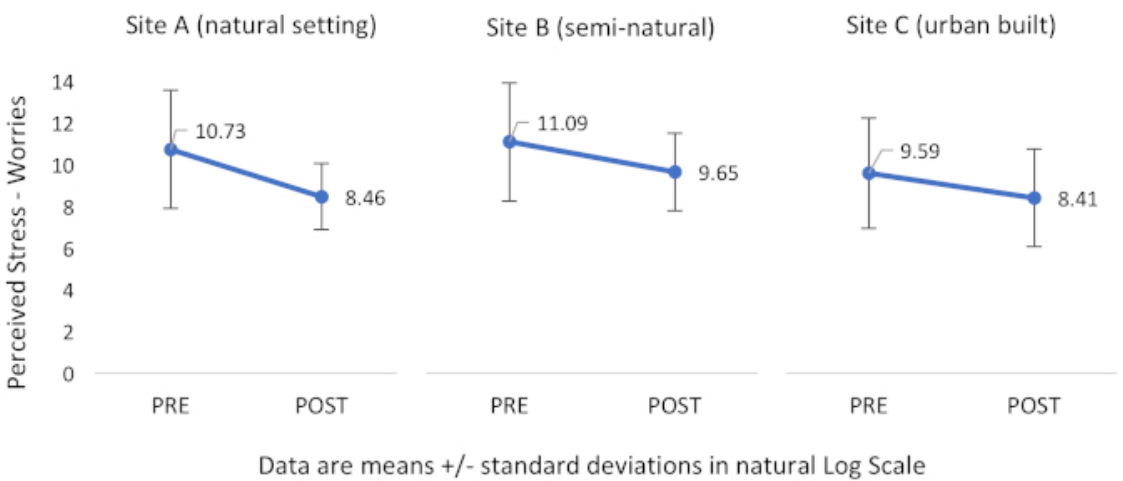

Figure 5: Changes in perceived levels of worries after visiting three different sites. This figure shows subjects' levels of worries decreased after visiting Sites $A, B$, and C. Data are presented as mean \pm SD. Please click here to view a larger version of this figure.

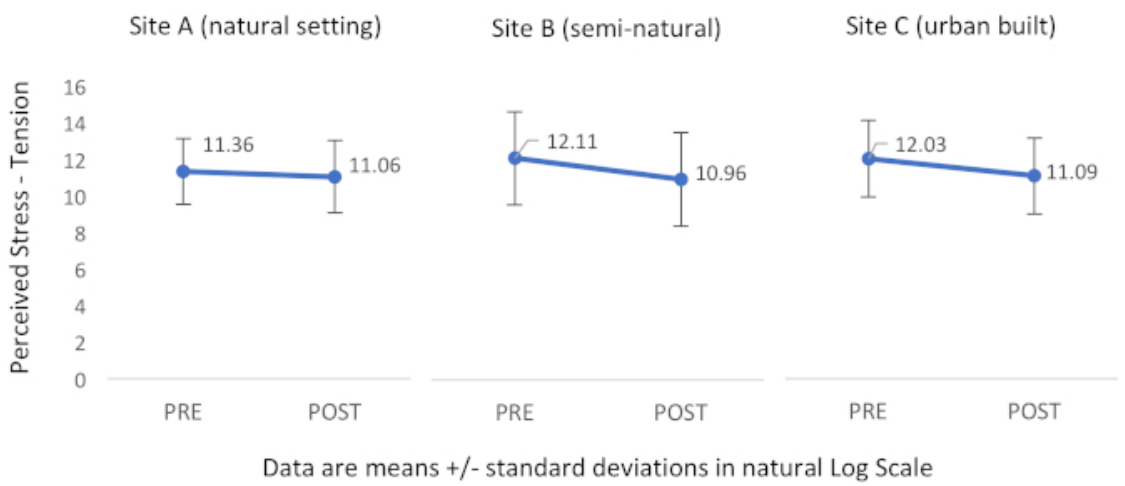

Figure 6: Changes in perceived levels of tension after visiting three different sites. This figure shows subjects' levels of tension decreased after visiting Sites $A, B$, and $C$. Data are presented as mean $\pm S D$. Please click here to view a larger version of this figure. 


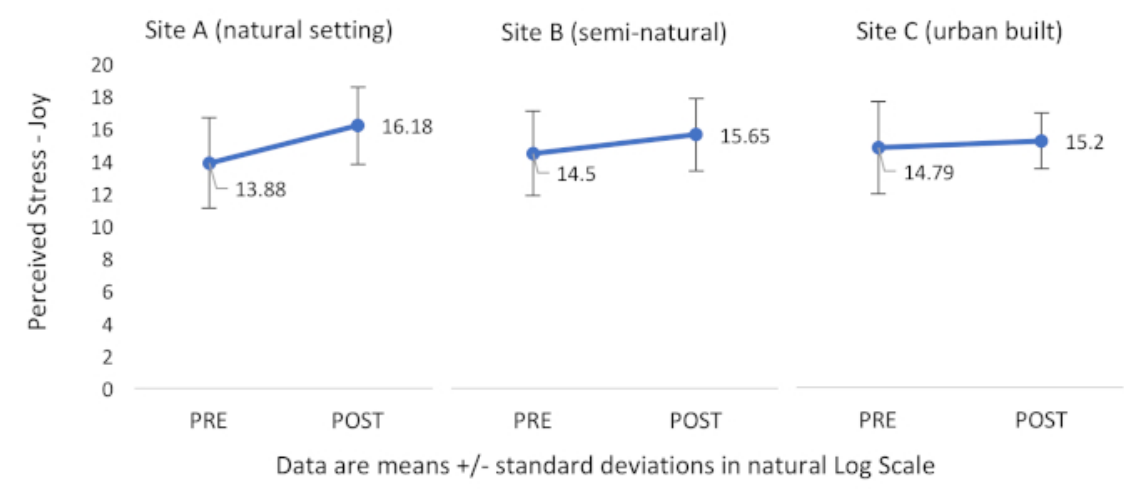

Figure 7: Changes in perceived levels of joy after visiting three different sites. This figure shows subjects' levels of joys increased after visiting Sites $A, B$, and $C$. Data are presented as mean \pm SD. Please click here to view a larger version of this figure.

\section{Discussion}

The aim of this study is to identify potential changes in stress using biophysical and psychological instruments following recreational visitation to three different settings with different levels of nature. Both cortisol and $\alpha$-amylase have been shown to be reliable indicators of levels of psychological stress. The amylase assay procedure described in this study has been adapted to a 96 well format. When amylase levels in saliva are high, absorbance changes occur rapidly. Therefore, it is critical to limit the number of samples analyzed at one time, as the number of samples that can be analyzed at one time is limited by how quickly $2.5 \mu \mathrm{L}$ of the sample can be added to each well. In this study, amylase levels were measured in one column (eight reactions) at a given time. Two principal systems are involved in the stress response, including the hypothalamus-pituitary-adrenocortical axis (HPA) and the sympatho-adrenomedullary system (SAM). Kirschbaum and Hellhammer ${ }^{14}$ have reported that salivary cortisol measurements are closely correlated with serum cortisol levels and involve less hyper-stress complications connected with blood sampling techniques. Alpha-amylase is a major salivary enzyme typically associated with sympathetic (SAM) stimuli ${ }^{15}$ and considered to be a useful measurement tool for evaluating the SAM system ${ }^{16}$.

While both the biophysical and psychological measures are efficacious in detecting changes in levels of stress, several issues are present in this study. First, there was incongruity between the cortisol and $\alpha$-amylase measurement outcomes, with differences noted at different sites. Nater et al. ${ }^{17}$ reported that there is little evidence as to whether the HPA (cortisol) or SAM (amylase) is predominant during psychological stress. One potential explanation was made by Takai et al. ${ }^{18}$, who suggested that the process by which $\alpha$-amylase and cortisol enter the bloodstream with cortisol, indicating that a more complex and lengthy system is in operation.

Another explanation of the results involves the level and saliency of stress ${ }^{17}$. The results suggest that acute levels of stress result in greater association between cortisol and a-amylase, and more moderate levels of stress result in greater disassociation. In this study, the stress experienced during recreational engagement are considered moderate at best. Thus, in studies such as the one presented here, in which experienced stress is low to moderate, differences in measured levels of cortisol and $\alpha$-amylase should be expected.

A third variable impacting the incongruity between the cortisol and $\alpha$-amylase measurements involves issues with salivary flow rate. There is limited evidence on the relationship between salivary flow rate, collection techniques, and stress ${ }^{19}$. In this study, saliva is collected via "drooling" into a test tube. Subjects are advised not to chew gum or eat before data collection, but how diligent they were in this study to these guidelines is unknown. Furthermore, using a salivette device may be more effective in collecting the required amount of saliva within a specified time. Nater et al. ${ }^{20}$ have suggested that there may be no differences in various biochemical characteristics based on whether cups or salivettes are used.

Finally, this study uses the "drool" method of collecting saliva as described by Granger et al. ${ }^{21}$. This method of collecting saliva has several advantages over other approaches but requires a competent, compliant, awake, and capable participant. As such, children under the age of six or elderly subjects are typically not considered suitable respondents. The advantages of this method include a large sample volume that facilitates assays for multiple markers and the fact that an unused sample can be frozen for future assays. In addition, the drool method minimizes effects from substances used to stimulate saliva flow such as chewing gum and drink mixes.

Another method for collecting data involves the use of cotton pledgets, where the saliva is absorbed by the pledget and expressed out of the cotton into a collection vial via centrigugation. Shirtcliff et al. ${ }^{19}$ issued a cautionary note stating that in certain situations, filtering saliva through cotton can cause interference in immunoassays. Other approaches include use of filter paper and hydrocellulose microsponges ${ }^{21}$. While each approach has specific advantages and disadvantages, given the sample size utilized in this study, the drool sampling method was chosen.

In conclusion, an accumulating body of research from a broad spectrum of disciplines suggest that natural environments can have positive effects on human health ${ }^{6,22}$. Typical of these types of settings include parks, greenspaces, gardens, and forested areas. Factors associated with these types of areas that are considered beneficial to health ${ }^{23}$ include improved air quality, heightened opportunities for physical activity and social contact, and enhanced feelings of quality of life. For example, Gidlow et al. ${ }^{24}$ found that while physical exercise had salutogenic effects in both natural and urban environments, natural settings were often more effective in reducing levels of stress.

Using a multiple-method approach involving biophysical measurements of cortisol and $\alpha$-amalyze and a self-report measuring perceived levels of stress, this study provides added support for the expanding body of literature suggesting that natural settings have beneficial effects on health- 
related issues such as reducing levels of stress ${ }^{25,26}$. This study also suggests that greater levels of nature have more pronounced potential benefits.

Several limitations exist in this study. The first is the fidelity of the biophysical data collection. While visitors were engaged in the saliva collection during similar times, namely mid- to late-afternoon through early evening, to account for the diurnal cycle of cortisol, researchers attempted to identify only those who had not eaten anything less than $2 \mathrm{~h}$ prior to collecting saliva. This was done via verbal questioning of when they had last ingested food. Thus, the researchers were dependent on the truthfulness of subject responses.

Second, because of the timing of data collection, response biases may have occurred within these samples, which may have been different if collection was done at a different time or by random selection. That is, subjects who visited each location in the afternoon or evening may not be representative of potential respondents who visited at different times.

Finally, while the biophysical data were collected and processed through well-recognized procedures, there was no measurement ascertaining chronic levels of stress. In this case, future studies should include a cortisol measurement using hair samples or similar techniques that determine long-term levels of stress before engagement in recreational activities at various locations.

\section{Disclosures}

The authors declare no conflicts of interest.

\section{Acknowledgments}

This study was funded in part through the Faculty Research Grant Program (FRGP) sponsored through the School of Public Health at Indiana University, Bloomington, IN. The authors would like to thank Dr. Alison Voight and Melissa Page for their editorial assistance and constructive comments.

\section{References}

1. Hansmann, R., Hug, S., Seeland, K. Restoration and stress relief through physical activities in forests and parks. Urban Forestry and Urban Greening. 6, 213-225 (2007).

2. Krantz, D. S., McCeney, M. K. Effects of psychological and social factors on organic disease: A critical assessment of research on coronary heart disease. Annual Review of Psychology. 53, 341-369 (2002).

3. Ward Thompson, C. et al. More green space is linked to less stress in deprived communities: Evidence from salivary cortisol patterns. Landscape and Urban Planning. 105, 221-229 (2012).

4. Haluza, D., Schonbauer, R., Cervinka, R. Green perspectives for public health: A narrative on the physiological effects of experiencing outdoor nature. International Journal of Environmental Research and Public Health. 11, 5445-5461 (2014).

5. Korpela, K. M., Ylen, M., Tyrväinen, L., Silvennomen, H. Determinants of restorative experiences in everyday favorite places. Health and Place. 14, 636-652 (2008).

6. Mantler, A., Logan, A. C. Natural environments and mental health. Advances in Integrative Medicine. 2, 5-12 (2015).

7. Mayer, F. S., McPherson-Frantz, C., Bruehlman-Senecal, E., Dolliver, K. Why is nature beneficial? the role of connectedness to nature. Environment and Behavior. 41, 307-643 (2009).

8. Pretty, J., Peacock, J., Sellens, M., Griffin, M. The mental and physical health outcomes of green exercise. International Journal of Environal Health Research. 15, 319-337 (2005).

9. Ulrich, R. et al. Stress recovery during exposure to natural and urban environments. Journal of Environmental Psychology. 11, 201-230 (1991).

10. Kaplan, S., Talbot, J. F. Psychological Benefits of a Wilderness Experience. In Behavior and the Natural Environment. Eds. Altman I., Wohlwill J. F., 163-203, Springer. Boston, MA (1983).

11. Salmon, P. Effects of physical exercise on anxiety, depression, and sensitivity to stress: A unifying theory. Clinical Psychology Review. 21, 33-61 (2001).

12. Focht, B. C. Brief walks in outdoor and laboratory environments: Effects on affective responses, enjoyment, and intentions to walk for exercise. Research Quarterly for Exercise and Sport. 80, 611-620 (2009).

13. Fliege, H. et al. The Perceived Stress Questionnaire (PSQ) reconsidered: Validation and reference values from different clinical and healthy adult samples. Psychosomatic Medicine. 67, 78-88 (2005)

14. Kirschbaum, C., Hellhammer, D. H. Salivary cortisol in psychoneuronendocrine research: Recent developments and applications. Psychoneuroendocrinology. 19, 313-333 (1994)

15. Gallacher, D. V.; Petersen, O. H. Stimulus-secretion coupling in mammalian salivary glands. International Reviews in Physiology. 28, 1-52 (1983).

16. Slosnik R. T., Chatterton, R. T., Swisher, T., Par., S. Modulation of attentional inhibition by norepinephrine and cortisol after psychological stress. International Journal of Psychophysiology. 36, 59-68 (2000).

17. Nater, U. M. et al. Stress-induced changes in human salivary alpha-amylase activity-associations with adrenergic activity. Psychoneuroendocrinology. 31 (1), 49-58 (2006).

18. Takai, N. et al. Effect of psychological stress on the salivary cortisol and amylase levels in healthy young adults. Archives of Oral Biology. 49 (12), 963-968 (2004).

19. Shirtcliff, E. A., Granger, D. A., Schwatz, E., Curran, M. J. Use of salivary biomarkers in biobehavioral research: Cotton based sample collection methods can interfere with salivary immunoassay results. Psychoneuroendocrinology. 26, 165-173 (2001).

20. Nater, U. M. et al. Human salivary alpha-amylase reactivity in a psychosocial stress paradigm. International Journal of Psychophysiology. $\mathbf{5 5}$ (3), 333-342 (2005). 
21. Granger, D. A. et al. Integration of salivary biomarkers into developmental and behaviorally-oriented research: Problems and solutions for collecting specimens. Physiology and Behavior. 92, 583-590 (2007).

22. Frumkin, H. Beyond toxicity: Human health and the natural environment. American Journal of Preventive Medicine. 20, 234-240 (2001).

23. Hartig, T., Mitchell, R., de Vries, S., Frumkin, H. Nature and health. Annual Review of Public Health. 35, $207-228$ (2014).

24. Gidlow, C. J. et al. Where to put your best foot forward: Psycho-physiological responses to walking in natural and urban environments. Journal of Environmental Psychology. 45, 22-29 (2016).

25. Ewert, A., Chang, Y. Levels of nature and stress response. Behavioral Sciences. 8 (5), 49 (2018).

26. Wyles, K. J. et al. Are some natural environments more psychologically beneficial than others? The importance of type and quality on connectedness to nature and psychological restoration. Environment and Behavior. 51 (2), 111-143 (2019). 